\title{
Adaptable legged-magnetic adhesion tracked wheel robotic platform for misaligned mooring chain climbing and inspection
}

\author{
Mahesh Dissanayake ${ }^{1}$, Tariq Sattar ${ }^{1}$, Shehan Lowe ${ }^{2}$, Ivan Pinson ${ }^{2}$, Tat-Hean Gan ${ }^{2}$ \\ London South Bank University, London ${ }^{1}$, TWI Ltd, Cambridge ${ }^{2}$
}

\begin{abstract}
Mooring chains used to stabilise offshore floating platforms are often subjected to harsh environmental conditions on a daily basis, i.e. high tidal waves, storms, etc. Chain breaking can lead to vessel drift and serious damage such as riser rupture, production shutdown and hydrocarbon release. Therefore, the integrity assessment of chain links is vital, and regular inspection is mandatory for offshore structures. Currently, structural health monitoring of chain links is conducted using either ROV's which comes at a high cost or by manual means which increases the danger to human operators. This paper presents a Cartesian legged tracked-wheel crawler robot developed for mooring chain inspection. The proposed robot addresses the misalignment condition of the mooring chains which is commonly evident in in-situ conditions. The mooring chain misalignment is investigated mathematically and used as a design parameter for the proposed robot. The robot is validated with laboratory based climbing experiments. The robot can be used as a platform to convey equipment, i.e. tools for nondestructive testing/evaluation applications.
\end{abstract}

\section{Key Words}

Mooring chain; Chain climbing robot; Magnetic adhesion legged robot; Tracked-wheel crawler; Inspection platform; Robot Design.

\section{Introduction}

An exponential increase of floating oil and gas production systems has been recorded around the world due to the high consumption and demand for fossil fuel energy. In 2013, 277 Floating Production Units (FPU) were recorded of which 62\% were reported as Floating Production Storage and Offloading (FPSO) units (Gordon, et al., 2014). With growth and advances in the shipping industry, it became necessary to maintain a floating structure within a given (prespecified) position/tolerance. Mooring chains were introduced for that purpose in 1808. These mooring chains experience harsh environmental conditions such as high tidal waves, storms, hurricanes, and the effect of salt water. It is therefore important to perform regular integrity 
assessment due to the significant damages which can occur and cause vessel drift, riser rupture, production shutdown and hydrocarbon release. As a result of such an event, the "Gryphon Alpha" spent over $\$ 1.8$ billion to resume operations after its mooring failure (Elman, et al., 2013). Between 2001 and 2011, 21 accidents were recorded, 8 of them reported as significant accidents (Kai-tung Ma, et al., 2013). Moreover, multiple mooring breakings can lead to catastrophic events which can be harmful to both humans and the environment. An FPU can suffer a mooring failure every 4.7 year (According to reported data from the North Sea between 1980-2001) (Angulo, et al., 2017). A single mooring failure, breakage or damage can cost approximately $£ 2 \mathrm{M}-10.5 \mathrm{M}$ to the operator (Noble Denton Europe Limited, 2016). Periodic inspection became mandatory for mooring systems (offshore floating platforms) after considering the potential human-related as well as environmental-related damages (Angulo, et al., 2017). Integrity assessment of offshore floating platforms needs to be addressed by providing in-situ physical access to the mooring systems as the removal and transportation of chain links for inspection/repair is not practical. Most of the reliable integrity assessment methods such as ultrasound testing (Rudlin, 2014), guided wave inspection, mechanical measurements, etc. require physical access to the chain to assess the structural health in in-situ conditions. Trained Non-Destructive Testing (NDT) divers and ROV inspections are the most common industrial mooring inspection methods. These methods raise health and safety concerns and diver inspection is very hazardous when inspecting a chain in the splash zone area ( Angulo, et al., 2017). Using ROVs is expensive and access to the chain is limited. Removing and replacing mooring chains for inspection is a costly and not very reliable method due to harsh operational conditions. Introducing an automated or teleoperated platform which can carry suitable NDT tools along in-service chain-lines will help to enhance the integrity management of mooring chains in in-situ conditions.

The development of chain climbing robots is still in its infancy due to the complicated climbing structure presented by mooring chains. The structure is discontinuous, curved and with uneven surfaces. Only a few robots/automated systems are reported in the literature, such as the 'MOORINSPECT' amphibious inchworm chain climbing robot presented in (Ruiz, et al., 2014) (Edwards, et al., 2013). This robot uses two arms to pull the robot structure up along the chain. With a long-range ultrasound guided wave collar and NDT deployment method, the robot weight in air is up to $750 \mathrm{~kg}$. In 2004, a human-like climbing robot was designed to inspect and clean anchor chains (Weiss, et al., 2004). An automated system to identify manufacturing stage defects (inspect welding joints on chain links) has been presented in (García, et al., 2004). 
A crawler type robot mechanism was presented in (Williams, 2008) with its locomotion assisted by gravity and a cable mechanism. Automated mechanisms /visual aided NDT measurements assisted by Remotely Operated Vehicles (ROVs) for subsea mooring chain inspection were also reported in the literature (Welapetage, 2017), (Yoshie \& Toshinari ,2013) and (Hall, et al., 1999).

Using NDT trained divers is the most common method of chain inspection but due to health and safety concerns divers are not permitted to access the entire chain (especially the splash zone area) (Angulo, et al., 2017). Visual inspection using ROV's is the next common method of NDT but ROVs are only able to operate under the sea level, and the splash zone cannot be inspected due to the limitations of the ROV. According to the history of mooring chain accidents and breakings, conventional ROV inspection cannot be considered as a reliable method (Kai-tung Ma, et al., 2013) (Angulo, et al., 2017). When considering the catenary curvature of a chain, chain link misalignments and offshore rough environmental conditions, deployment of large /heavy robots is not convenient (Angulo, et al., 2017). Most of the robotic platforms are designed for diver deployment with mechanical lifting supports. Handling considerably large weights manually in operational conditions is not easy or safe. Therefore, additional deployment tools and supports are needed. When considering the above-mentioned state-of-the-art chain inspection/climbing mechanisms, development of a new lightweight robotic mechanism/platform which can climb mooring chains both in air and water is needed.

This paper describes the design and prototyping of a lightweight, permanent magnetic adhesion, tracked-wheeled robot which can be easily deployed. The primary purpose of the presented robotic platform is to convey NDT equipment along the chain to perform an inspection. The preliminary design of the proposed robotic platform was previously published by the current authors in (Dissanayake, et al., 2018) and (Dissanayake, et al., 2017). This paper describes an upgraded version of the robotic platform which solves the climbing problem posed by link misalignment of mooring chains. The previously published magnetic adhesion robotic platform was able to climb along orthogonal chain links which are uniformly in a straight line, but it was unable to adapt to chain curvature and chain link misalignment due to relative twists between links. This paper contains a brief description of the previously published robot and gives a mathematical illustration of the proposed misalignment adaptation in the first section. This is followed by the design of the proposed misalignment adaptation mechanism. The final section of the paper describes the prototype and validation of the climbing technique. 


\section{Understanding of misalignments and tracked-wheel orientations}

During the previous research developed a climbing robot for a vertically aligned chain and was presented as a platform to convey tools required to conduct in-service activities i.e. structural health monitoring. An orthogonally positioned (refer to figure1) magnetic adhesion tacked-wheel robotic approach was used in this research. Tracked-wheel units were rigidly attached to the main body of the robot and research was conducted to test the capability of climbing when chain links are strictly orthogonal to each other (ideal laboratory condition). The magnetic adhesion module, motor requirement calculation, structural integrity study and prototype of the tracked-wheel units were reported in the previous study (Dissanayake, et al., 2018).

(a)

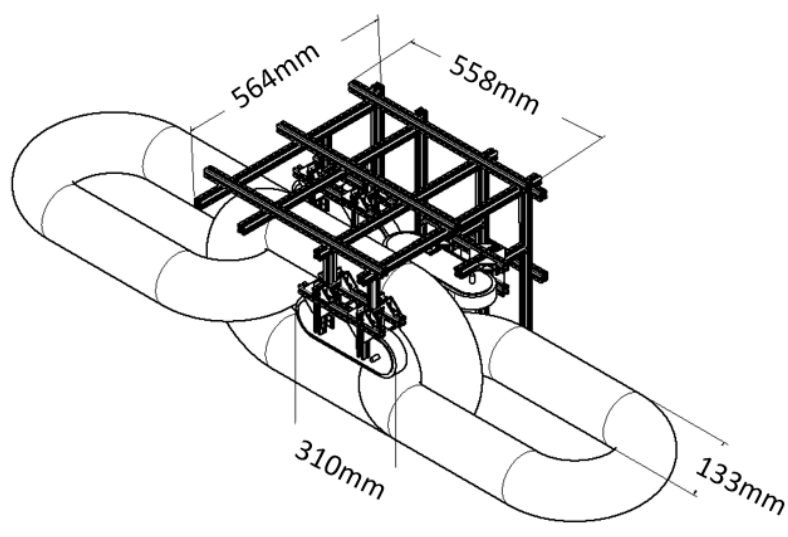

(b)

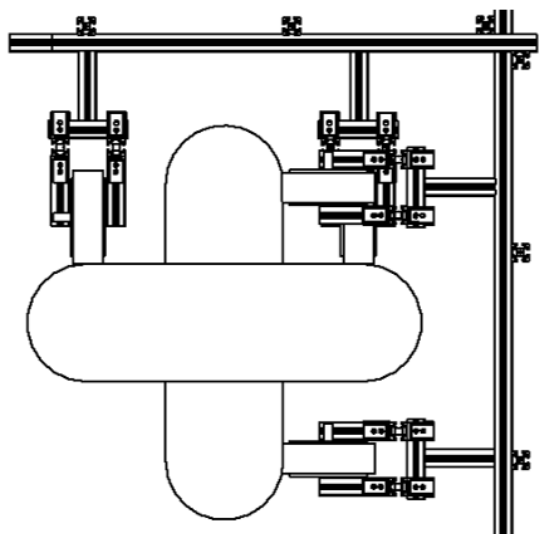

Figure 1 - orthogonally positioned magnetic adhesion tracked-wheel climbing robot. (a) Climbing robot design. (b) Orthogonal tracked wheel placement Concept: - (Dissanayake, et al., 2018)

Due to in-situ environmental forces, chain links are not always orthogonal to each other. The magnetic adhesion tracked-wheel crawler robot requires a good surface contact to generate a sufficient friction force for climbing. When there is a misalignment in the chain link orientation, the robot is unable to adapt its orientation due to the rigid body attachments. Further investigations were conducted to understand the behaviour of the tracked-wheel module with respect to selected misalignments. Two types of chain misalignments were considered in this study (common misalignments when the chain is hanging vertically). Figure 2, illustrates an ideal (non-misaligned) chain link. To ease the visualisation, the chain surface in figure 2 -a is plotted as a planar surface. Due to the curvature of the mooring chain links, the centre of the chain link was selected as the optimum crawling path as illustrated in figure 2 -a. To place the 
tracked-wheel unit on the optimum crawling path, a pure translation needs to be applied (refer equation 01 and figure $2-b)$.

$P($ ideal $)=P($ home $)+\left(\begin{array}{c}d 1 \\ -d 2 \\ 0\end{array}\right)$

where $\mathrm{P}$ (ideal) represents the optimum crawling position when the chain links are in ideal conditions. In order to generalise the tracked-wheel positions, the ideal path is not taken as the home position. $d 1, d 2$ are directional distances (refer figure 2-b) form a given home position (home - considered as the edge of the frame in this case).

(a)

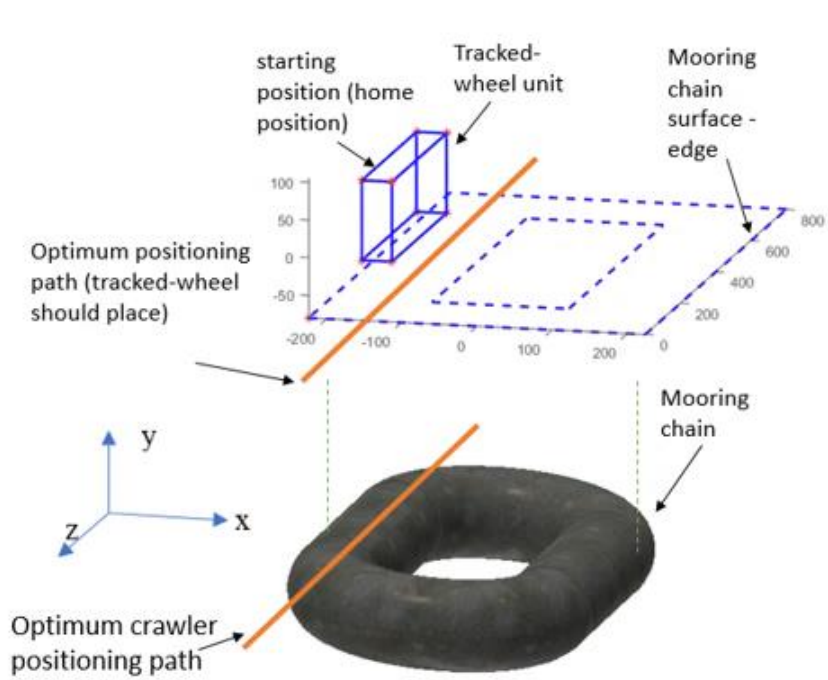

(b)

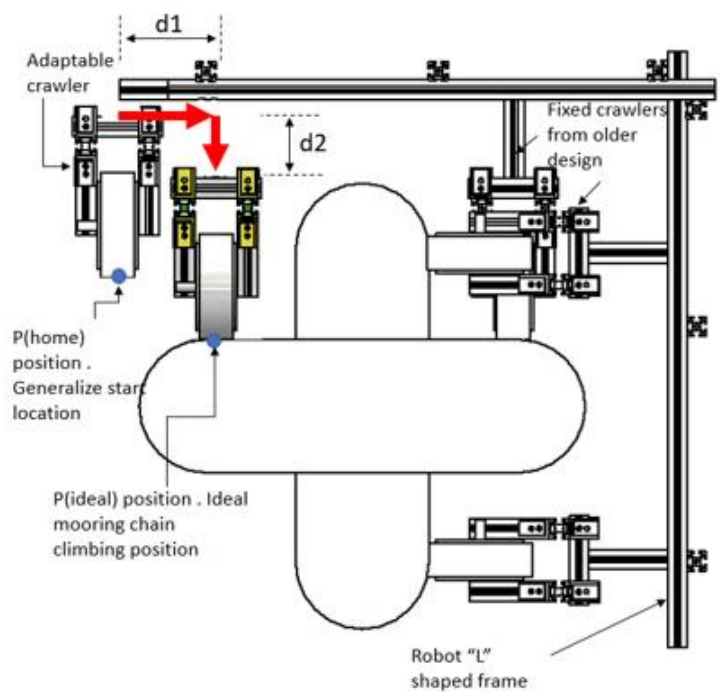

Figure 2-(a)-Model of a chain link without misalignments. (b)- Placement distances

The first misalignment is explained in figure 3-a. A chain link is rotated around its $\mathrm{z}$-axis with an angle of $\alpha$ (angle measured with respect to the $\mathrm{x}$-axis). To place the crawler on the chain in the same way as the ideal scenario, the wheel unit should be translated on to the new point in the $x-y$ plane (refer figure 3-b). Due to the tangential placement of the wheel unit as illustrated in figure 4, it is not necessary to rotate the wheel unit (rotation to cope with the twist angle) when there is a twist type misalignment. If this feature is not considered, another degree of freedom has to be added to the system, i.e. rotation around the wheel unit's z-axis. Therefore, $\mathrm{p}\left(\right.$ twist $\left._{\mathrm{z}}\right)$ can be written as follows,

$P\left(\right.$ twist $\left._{z}\right)=P($ ideal $)+\left(\begin{array}{c}\Delta x \\ \Delta y \\ 0\end{array}\right)$ 
where, $P$ (twist $\mathrm{z}$ ) is the optimum crawling position when the chain link is in misaligned condition (rotate around the chain links $\mathrm{z}$ axis). $\Delta \mathrm{x}$ and $\Delta \mathrm{y}$ are directional distances (refer figure 3-b) that the tracked wheel should move in order to cope with the misalignment.

(a)

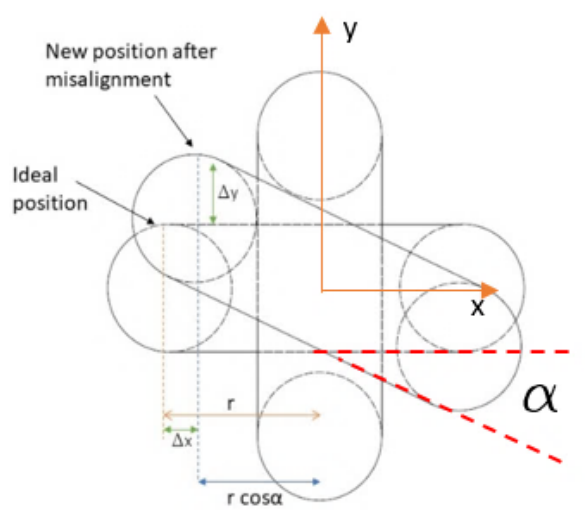

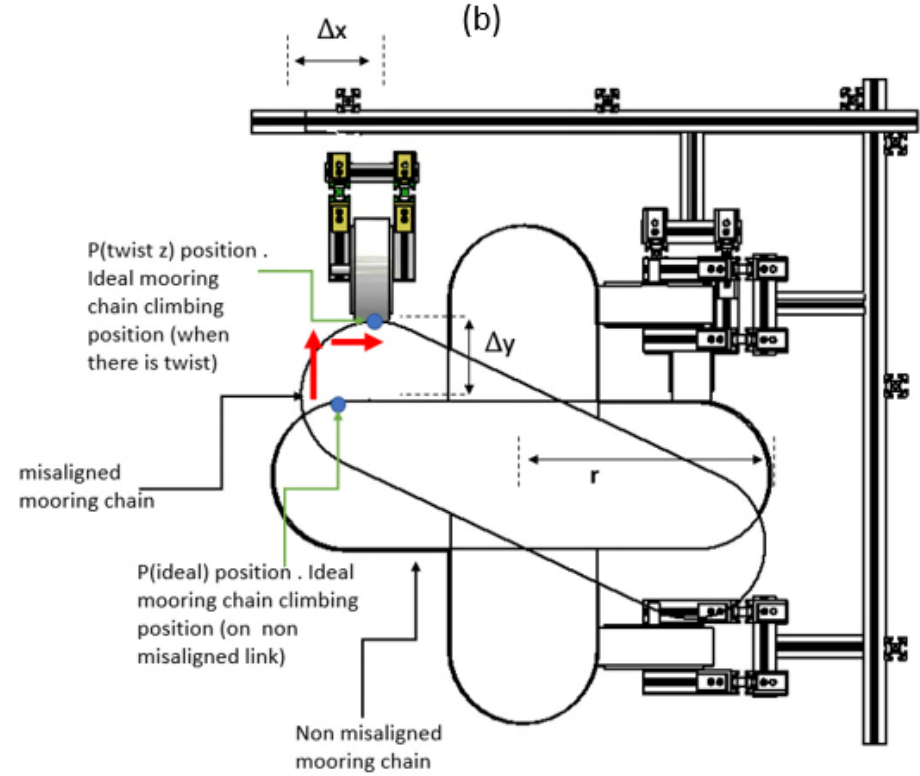

Figure 3-Misalignment case 01: (a) schematic of chain link rotated around the zaxis, (b) racked-wheel unit placement after introducing the translation

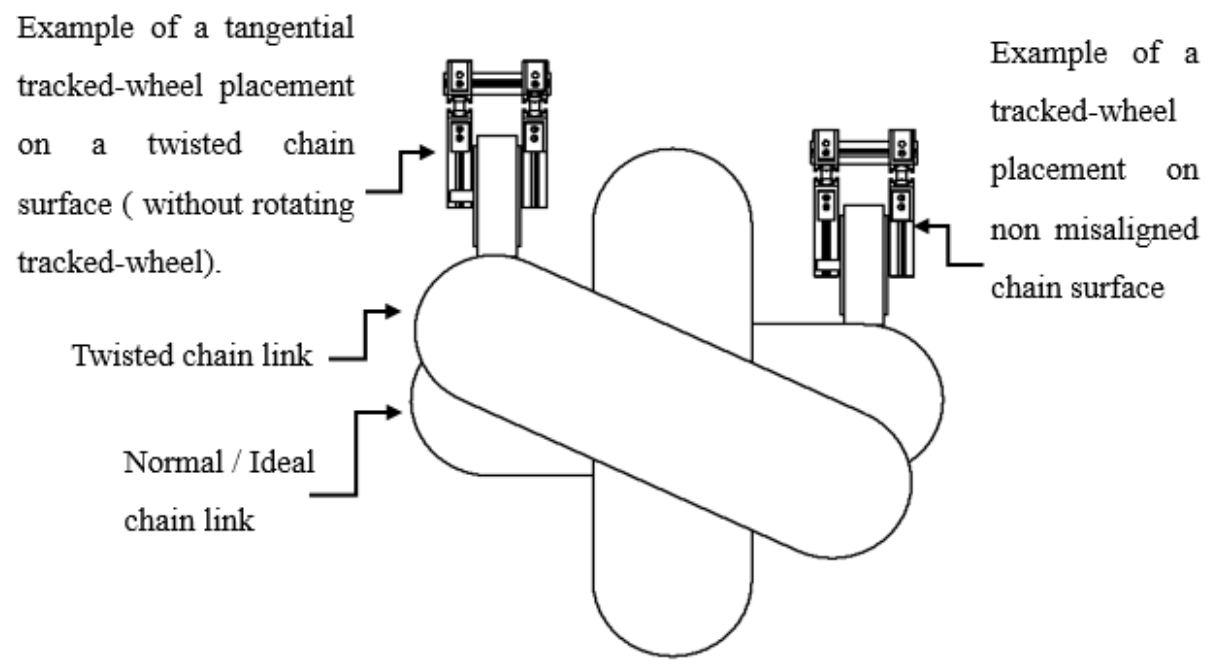

Figure 4 -tracked-wheel unit placement during misalignment

In the second misalignment explained in figure 5-a, a chain link is rotated along the $\mathrm{x}$-axis. To place the tracked-wheel unit on the chain surface, it is necessary to introduce a pure rotation to the $\mathrm{x}$-axis of the wheel unit. After applying the rotation, it is possible to create the appropriate angle which will allow a better surface contact (Refer figure 5-b). In this case, the tracked- 
wheel unit is already aligned in the optimum working axis. Therefore, no translation is needed. The robot's vertical climbing motion will be smoother after achieving this rotation. The required rotation can be modelled by $\mathrm{Eq}(3)$. Where, $\beta$ is the misaligned angle (refer figure 5-

b)

(a)

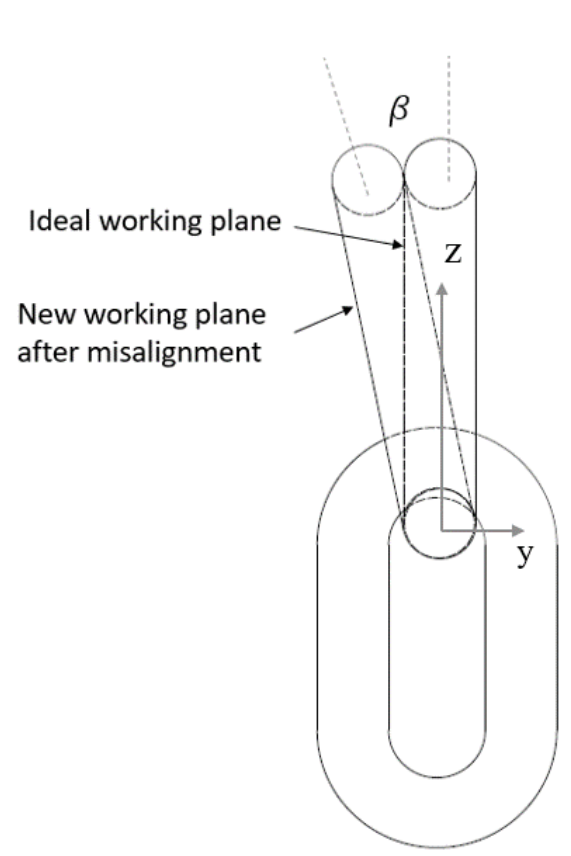

(b)



Figure 5-Misalignment case 02: (a) schematics of chain link rotates around the $x$-axis, $(b)$ tracked-wheel unit placement after introducing the rotation

$P 1\left(\right.$ twist $\left._{x}\right)=\left(\begin{array}{ccc}1 & 0 & 0 \\ 0 & \cos \beta & -\sin \beta \\ 0 & \sin \beta & \cos \beta\end{array}\right) \times P 1$ (ideal)

When observing the above 3 cases (ideal alignment, chain twist ( $\alpha$ ) and tilt ( $\beta$ ) misalignments), where $\alpha$-twist angle, $\beta$ tilt angle can be identified as the only variables which are required for wheel manipulation. The remaining variables are calculated with the help of angle variations (discussed later in the article). According to $\mathrm{Eq}(1-3)$, two translations and a rotation can be observed. Therefore, by introducing 3 degrees of freedom to the existing tracked-wheel crawler, adaptations can be made to the above mentioned misalignments. .

\section{Robotic manipulator design}

\subsection{Design of the 3DOF}

The three degrees of freedom, which are mentioned in the above section can be categorised as two translations (along $\mathrm{x}$ and $\mathrm{y}$-axis) and a rotation around the $\mathrm{x}$-axis. The above description is related to an operation of a planar Cartesian arm/leg with a revolute joint as an end effector. 
Modification capability of the existing design was also considered during the conceptual design. Moreover, two translations are modelled with prismatic joints, and the rotation is modelled with a revolute joint (refer figure 6). In the conceptual manipulator diagram (figure 6), L1 and L2 are link lengths due to possible mechanical attachment clearances. L3 is the distance between crawler attachment and the revolute joint. Variable parameters of two linear motions (prismatic joints) and revolute joint are $\mathrm{d} 1, \mathrm{~d} 2$ and $\emptyset$ respectively. Therefore, active transformation of the end-effector (refer to point ' $\mathrm{P}$ ' in figure 6-c) from it's home position (figure 6-a \& b) to its current position (with the given joint variables) can be expressed as $\mathrm{Eq}(4)$.

$$
(p)_{\text {Active transformation }}=\left(\begin{array}{cccc}
1 & 0 & 0 & L 1+d 1 \\
0 & \cos \emptyset & -\sin \emptyset & -d 2-L 2-L 3 * \cos \emptyset \\
0 & \sin \emptyset & \cos \emptyset & -L 3 * \sin (\emptyset) \\
0 & 0 & 0 & 1
\end{array}\right) \quad \mathrm{Eq}(4)
$$



(c)

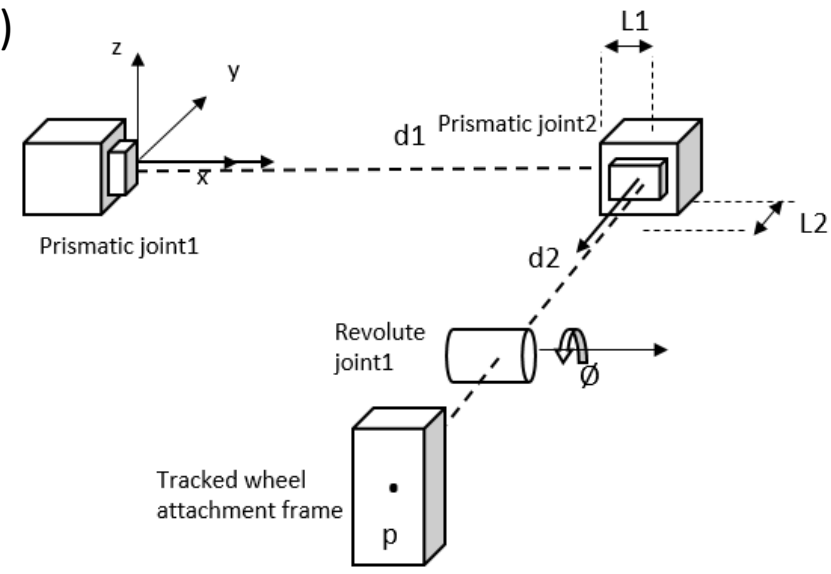

Figure 6 - Conceptual design of the robotic manipulator (schematics). (a) Home configuration explanation. (b) home configuration. (c) active transformation

\subsection{Robotic leg design for proposed kinematics motion .}

The rigidity of the leg design/mechanism is vital due to the mooring chain's rough and robust nature. Moreover, load/weight acting along the axis should be considered during the design of the above mechanism. To create a linear movement along the axis, DC geared actuators were used. The possibility of introducing a slider actuator was considered, but due to the load capacity and rigidity, a low friction dry coupled glider was introduced to the system with actuators as illustrated in figure 7. Two linear actuators were introduced to the system to make the tracked-wheel attachment rigid and stable. Dual actuator design with a pin type joint was introduced to achieve the pitch action (rotate along the $\mathrm{x}$-axis) of the wheel unit. The system 
strength can be enhanced by replacing the rotary axis with two actuators which create a pitch angle by changing the distance of each actuator. Both y-axis actuators are mounted on a linear glider. Bodies of ' $b$ ' actuators in figure 7 are mounted on a linear glider ' $c$ ', and both actuators are connected to by a rigid attachment " $\mathrm{f}$ ". The rigid attachment ' $\mathrm{f}$ ' is connected to the end of the actuator stroke ' $a$ '. The magnetic adhesion tracked wheel unit ' $e$ ' is attached to the actuator end by using a pin-type joint ' $\mathrm{d}$ '. Movement along the $\mathrm{x}$-axis is achieved by manipulating the actuator ' $a$ ' and $y$-axis movement can be made by using the actuator ' $b$ '. By introducing a differential motion to the ' $\mathrm{b}$ ' actuators, rotation along the $\mathrm{x}$-axis can be made. This actuator assisted robotic leg is mounted on the " $L$ " shaped main frame of the robot.

(a)

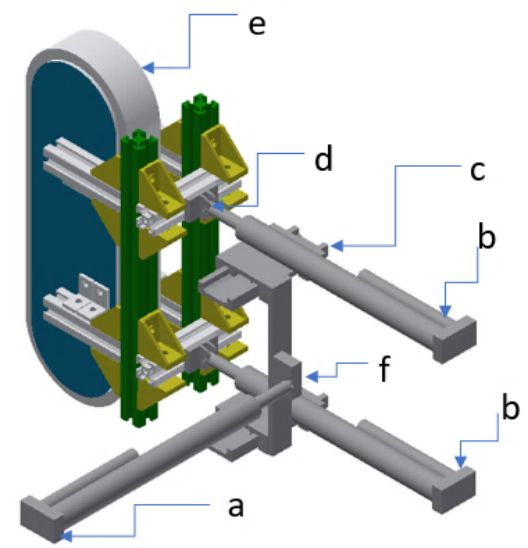

(b)

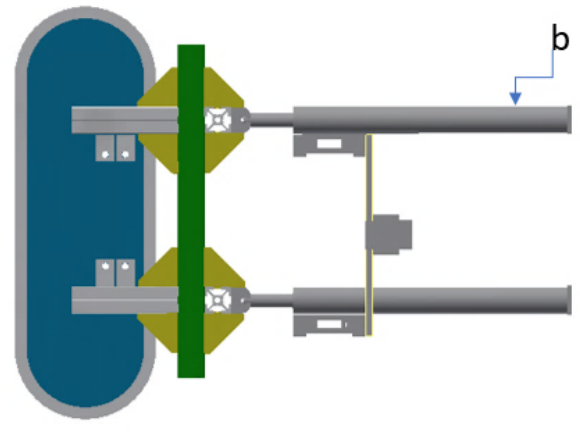

(c)

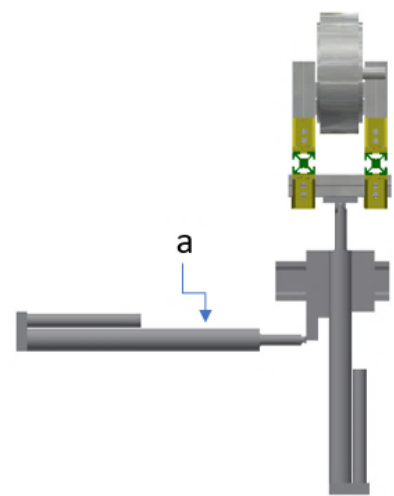

Figure 7 -Design of the robotic manipulator. a-design. b-planner mode. c-cartesian mode

\subsection{Kinematics of the actuators assisted robot leg}

In order to understand the kinematics of the design, entire motion of the leg can be separated into two main motions; planar manipulator (figure 7-b) and Cartesian manipulator (figure 7-c). The Cartesian manipulator operates as discussed in Section 3.1. For this actuator ' $a$ ' represent the $\mathrm{x}$-axis and both ' $\mathrm{b}$ ' actuators represent the $\mathrm{y}$-axis without any differential motions as illustrated in figure 8. Therefore, the active transformation of the wheel units (refer to point ' $\mathrm{P}$ ' in figure 8-c) in the 3D plane relative to the home configuration (figure 8- a \& b) can be expressed as $\operatorname{Eq}(5)$ where L1, L2, L3 are attachment distances and d1,d2 are variable actuator stroke lengths. It is possible to find the new translation point along the XY plane (refer to figure 8) with respect to the chain misalignment angle and known parameters of the chain link (refer figure 3-a). ' $r$ ' ( $r$ in figure 3a-b) is the distance between the optimum working path to the centre of the chain link. For a given chain 'r' will always be a known parameter. If the chain twist along the $\mathrm{z}$-axis is $\alpha, \mathrm{d} 1=\mathrm{r}-\mathrm{r} \cos (\alpha)$ and $\mathrm{d} 2=\mathrm{r} \sin (\alpha)$. 


$$
(p)_{\text {Active transformation }}=\left(\begin{array}{cccc}
1 & 0 & 0 & L 1+d 1 \\
0 & 1 & 0 & -(L 2+L 3+d 2) \\
0 & 0 & 1 & 0 \\
0 & 0 & 0 & 1
\end{array}\right)
$$
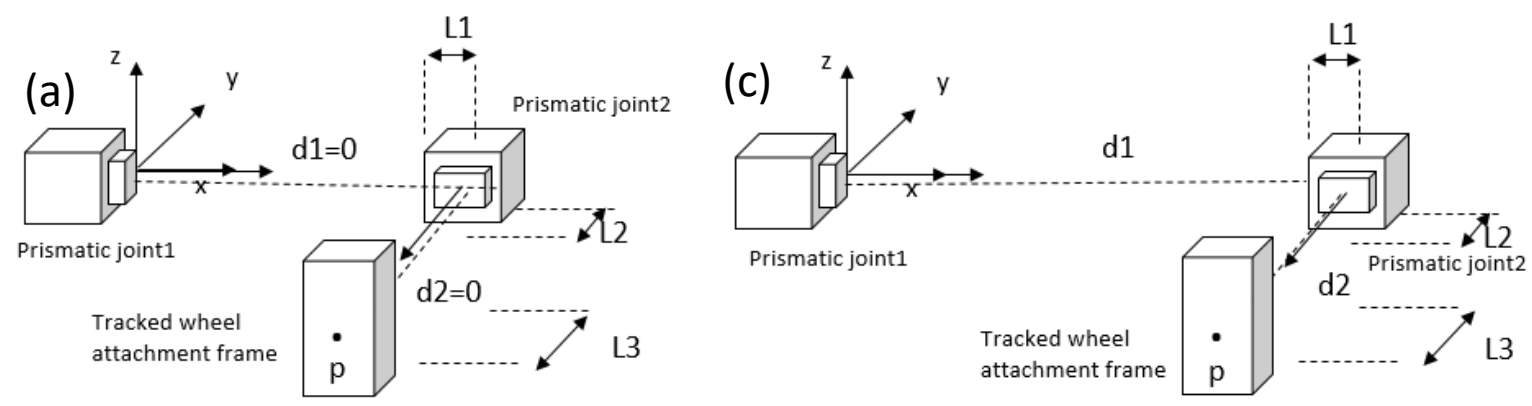

(b)

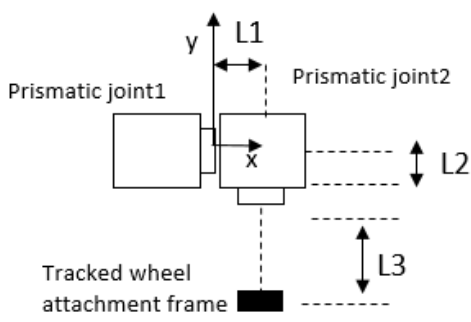

Figure 8 - Schematics of the for chain twist adapt operation. (a) Home configuration explanation. (b) home configuration. (c) active transformation

To understand the behaviour of the planar part of the robot leg, the differential motion of the 'b' actuators are considered (refer to figure 9). d2-1 and d2-2 represent variable stroke distances of the ' $b$ ' actuators. L2 and L3 are considered as fixed offset distances due to the mechanical design of linear actuators. D is the fixed vertical distance between 2 actuators.

$(\Delta \mathrm{d})$ differential distance $=\operatorname{distance}(d 2-2)-\operatorname{distance}(d 2-1)$

$\mathrm{Eq}(6)$

$(\Delta \mathrm{d})=\frac{D}{\tan (\varnothing)}$

$\mathrm{Eq}(6)$ and $\mathrm{Eq}(7)$ were used to calculate the appropriate actuator stroke distances. The sign of the angle was used to identify the associated actuator. For example, if the angle is (+ve), d2-2 actuator extended by Dd and d2-1 actuator kept as it is.

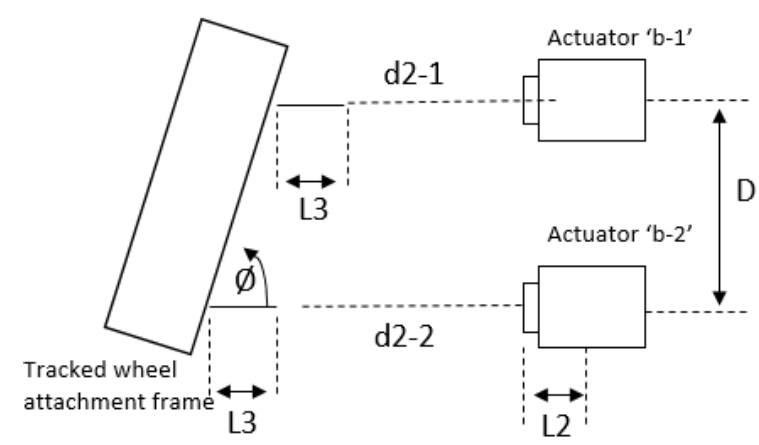

Figure 9 - Schematic of the for chain tilt adapt operation 


\subsection{Mitigating the misalignment}

The previously discussed legged magnetic adhesion tracked-wheel module was used to replace the rigid attachment (refer figure 10) to deal with the misalignment. Some unmodified legs of the robot are shown in figure 10-a to distinguish the modifications in the other figures. Potential NDT attachment places are marked in the figure 10 -a and 10-d, but the NDT system is not reported in this paper. Performance of the upgraded design was tested by using CAD models of chain twist and chain tilt misalignment scenarios (refer to figure 11).
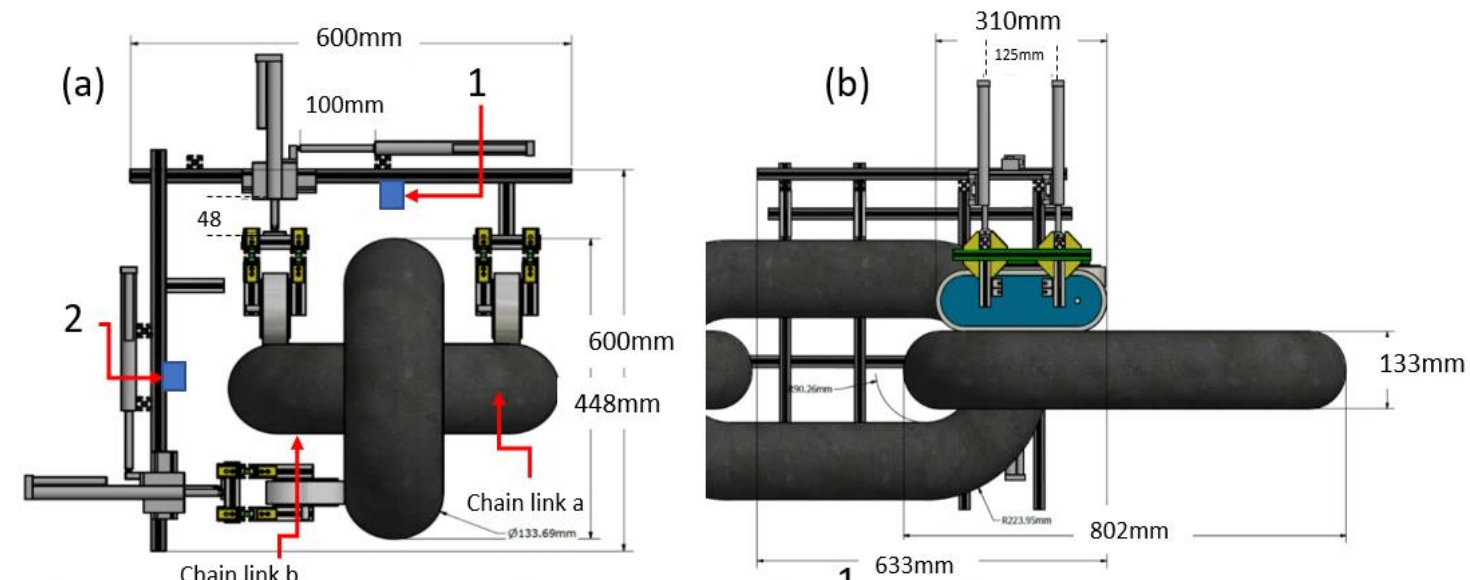

(c)
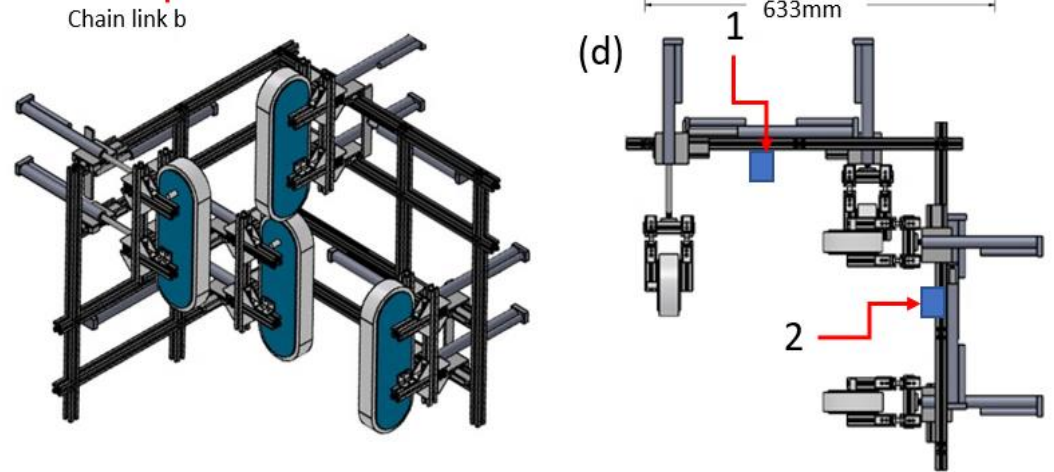

1,2 Potential places to attach a NDT equipment i.e., Camera , UT manipulator etc.,. 1 is to inspect chain link-a. 2 is to inspect chain link-b

Figure 10 - Robot $a \& b$ - design schematics $c \& d$-full design



(b)

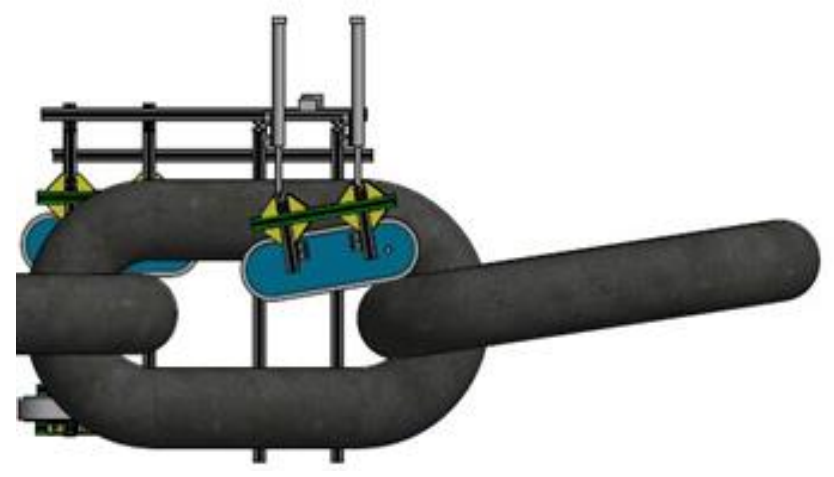

Figure $11-C A D$ compatibility of design. (a)twist adaptation. (b)tilt adaptation 


\section{Prototype and testing}

\subsection{DOF Cartesian leg}

A prototype of the Cartesian leg mechanism was developed to the design specifications provided in the previous section as illustrated in figure 12-a. The prototyped system in figure 12-a was attached to the robot's mainframe (L frame), and a 3rd linear actuator was added as illustrated in figure 12-b. Actuators were attached to the system by using a linear slider plate. The motion of the legged system was tested to observed 3DOF movement as explained in the design process (Eq 2-3). In order to test the 3DOF degree of freedom, the proposed movements were observed as illustrated in figure 13 without attaching the robot to a chain. During the development of the prototype, 5-8mm clearance was introduced to the pin joint for mechanical advantage during the tilt motion.

Linear glider

attachment

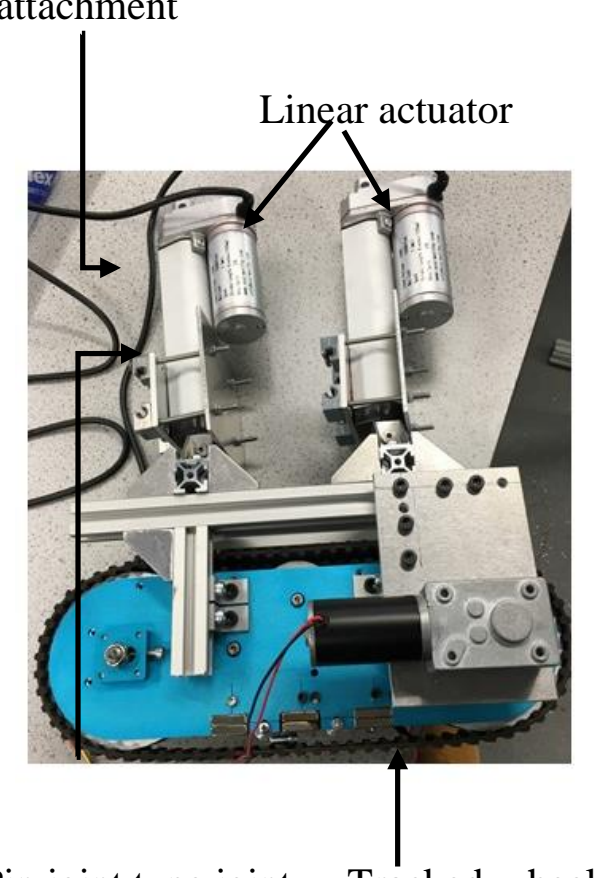

(a)

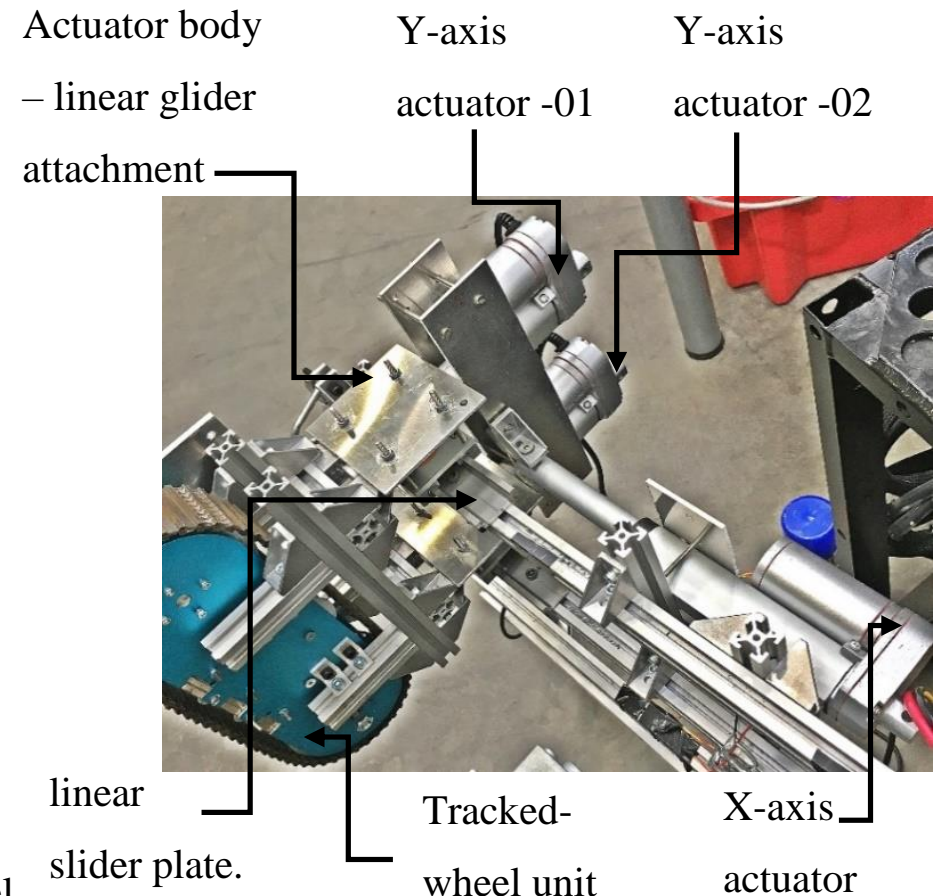

Pin joint type joint Tracked-wheel

(b)

Figure 12 - Prototyped Cartesian legged tracked-wheel unit. (a) prototyped robot leg with 2 actuators. (b) robot leg mounted on the robot with the 3rd actuator 


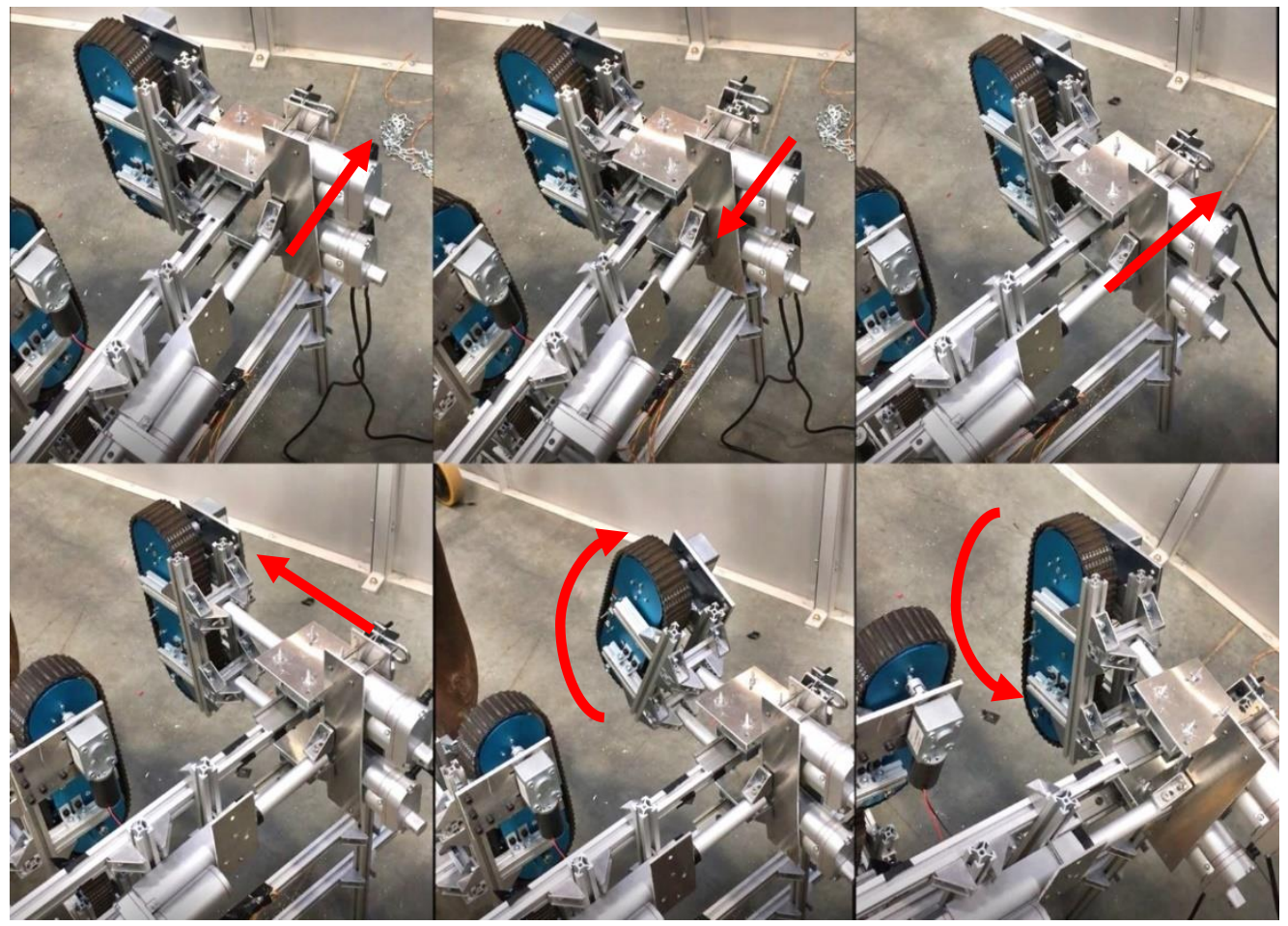

Figure 13 - Prototyped Cartesian legged 3DOF testing

\subsection{Climbing test}

To test the Cartesian legged motion of the robot, link tilt and link twist misalignments were introduced to a 3-link chain segment as illustrated in figure 14 and climbing capability was tested. To create the mooring chain twist misalignment, a series of wooden wedges were inserted in between the first and second links. A tensioned strap was used as illustrated in Figure 14 to create tilt misalignment.

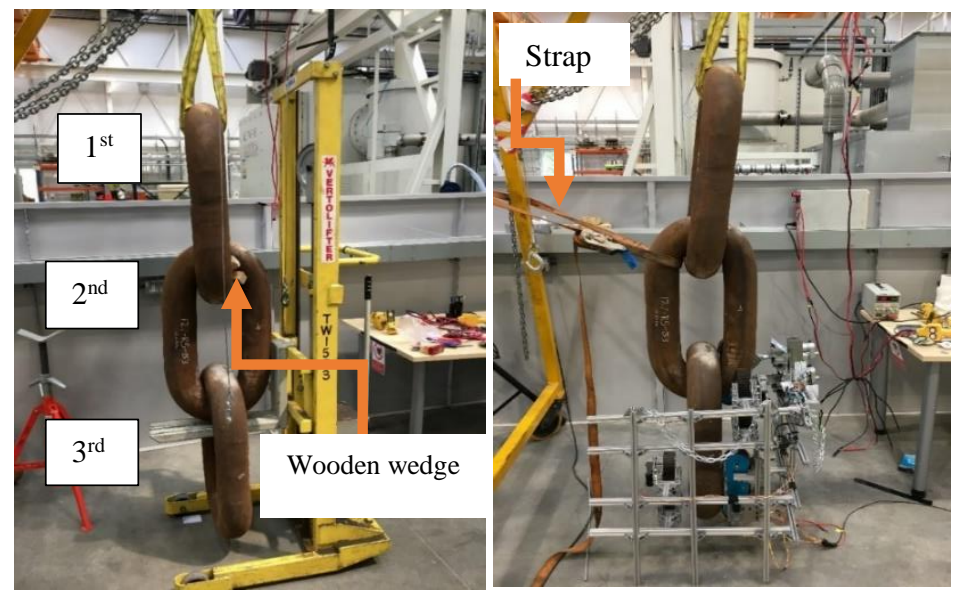

Figure 14 - Robot climbing sequence testing test rig (misalignment test rig) 
Chain twist misalignment was introduced to the 1 st link as illustrated in the above experimental setup and climbing capability was tested as illustrated in figure 15 . The robot was able to adapt to the misalignment of the chain link and was able climb along the chain. The robot was also able to adapt and climb when a tilt misalignment was introduced to the link 1 as illustrated in figure 16. A stability check was performed without safety cables (Figure 17). According to the experimental results, the robot stayed attached to the misaligned chain link surface with its own weight (all the safety cables were released during the stability test experiment).


Figure 15 - Robot climbing sequence testing for 10 deg. twist misalignment
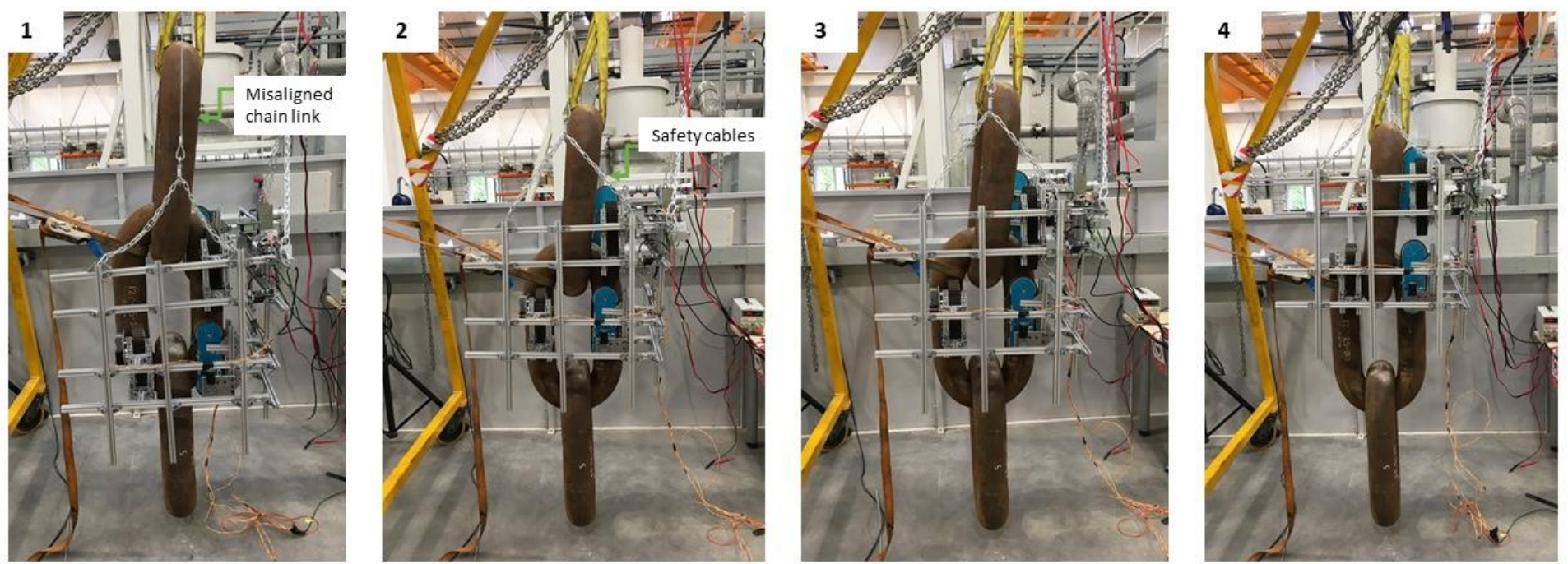

Figure 16 - Robot climbing sequence testing for 5 deg. tilt misalignment 


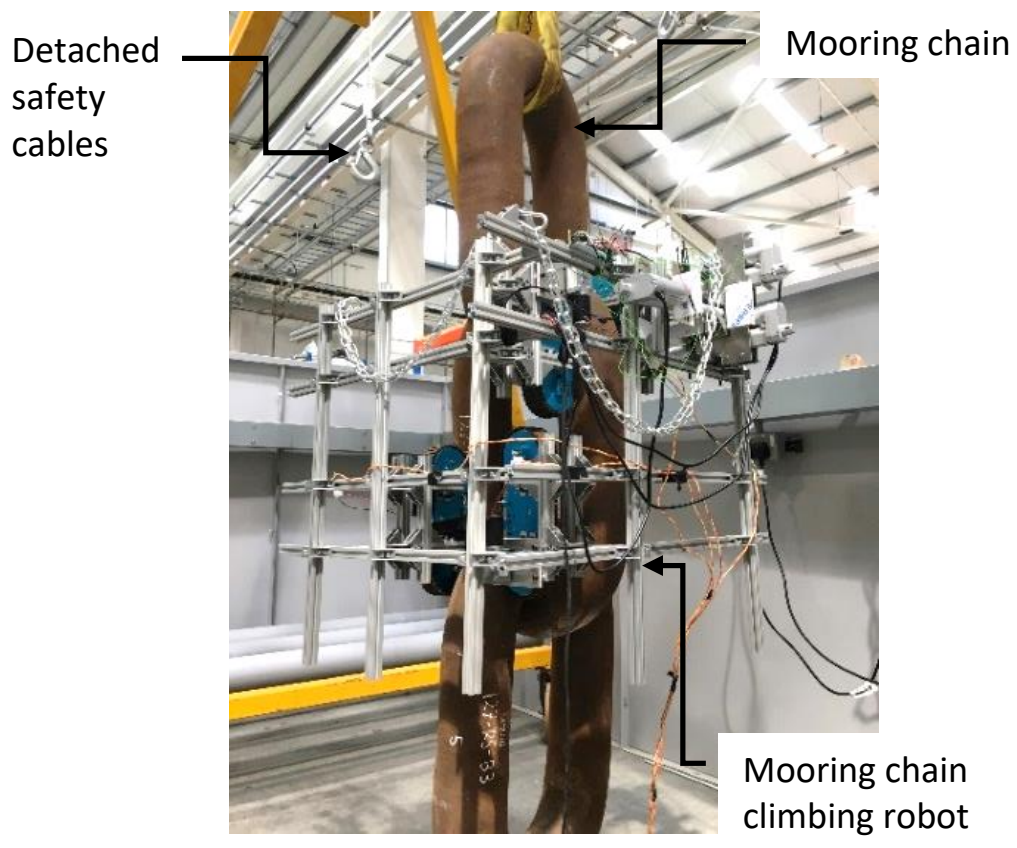

Figure 17 - Robot climbing stability check without safety cables

Actuator distances were recorded during the misalignment climbing and checked against the distances measured during the CAD study (refer to figure 19). There was a good agreement between the CAD distance measurements and experimental readings. The control architecture presented in figure 18 was used in this study for robot control. Automated detection of misalignment angles was not established within this study. Therefore, misalignment angles and control commands were added to the system as an input from the robot control GUI (LabVIEW), i.e. the commands Climb up, Climb down and the misalignment angles. The GUI was connected to a microcontroller (on the robot) via serial communication.

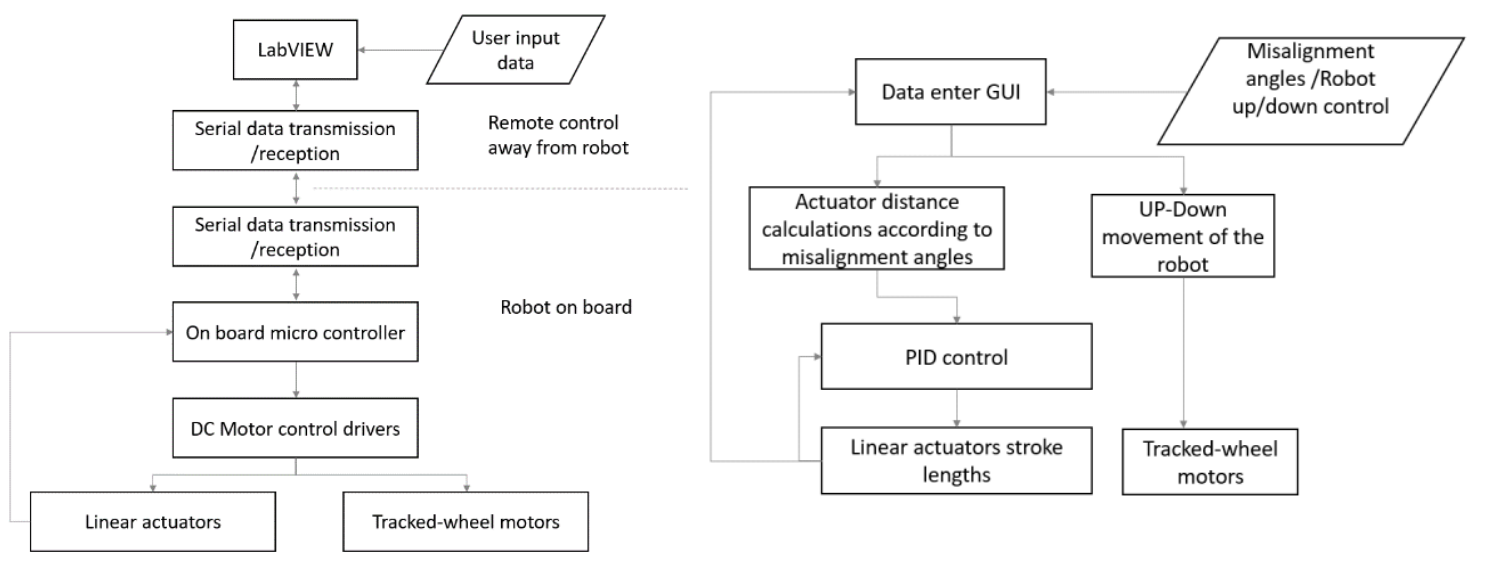

Figure 18:(a) hardware architecture diagram. (b) control flow chart 
(a)

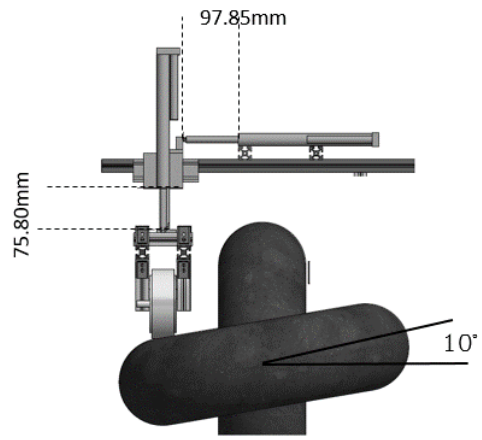

(c)

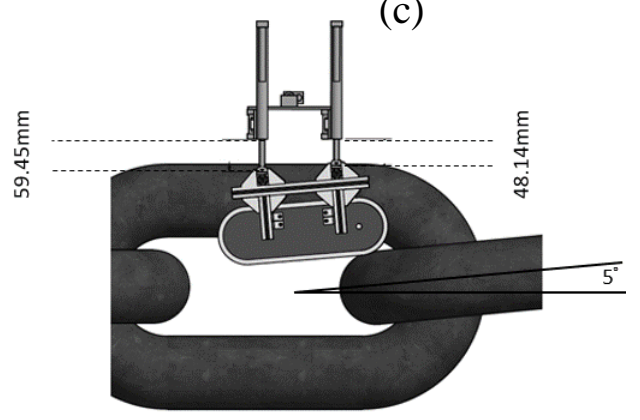

(b)
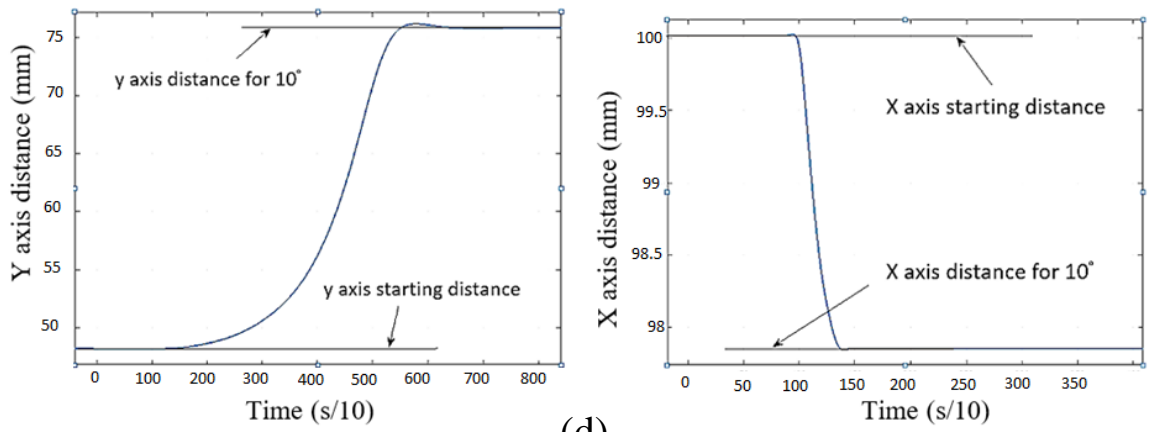

(d)



Figure 19 - Actuator distance check. (a) 10-degree twist misalignment CAD distances. (b) 10 degrees recorded actuator distances. (c) 5-degree twist misalignment CAD distances. (d) 5 degrees recorded actuator distances.

\section{Further improvements}

\subsection{Robot operational improvements}

Introducing multi legged robotic locomotion will be the main further improvement of this study (refer Figure 10-c-d). It is necessary to introduce an active control mechanism that can correct the robot when it starts slipping or slightly changing its path due to external forces or mooring chain surface issues. Mooring chains are amphibious structures and the robot is required to travel both in air and underwater. Therefore, it is necessary to use underwater motors and controllers. In the current study, misalignment angles were added to the system mechanically. Further work will add an automated misalignment angle detection system. 


\subsection{Chain misalignment detection feasibility study (numerical modelling and design)}

The misalignment detection of the proposed robot was conducted by manual measurement, and a numerical feasibility study was conducted to improve the robot by having autonomous misalignment detection capabilities. Sample misalignment angles (as discussed in the previous section) were introduced to a CAD file, and a FEA study was carried out by using COMSOL numerical modelling. The Finite Element Analysis (FEA) study was carried out by considering air and solid objects. COMSOL pressure acoustics and solid mechanics modules were incorporated to detect the misalignment using ultrasonic distance measurement transducers. The boundary load force on the transducer point was used as the excitation. Parameters considered in the FEA are tabulated in table 1 and model layout is presented in figure 24. A study of chain twist misalignment detection was carried out as illustrated in figure 20.

(a)

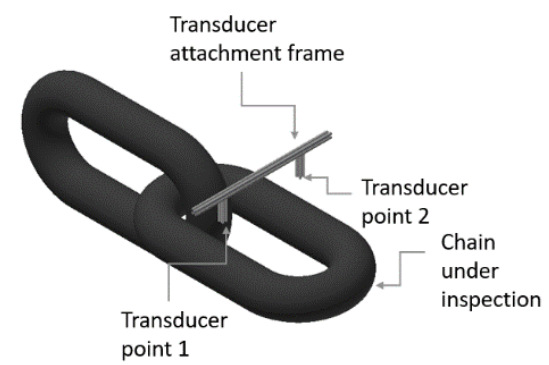

(b)

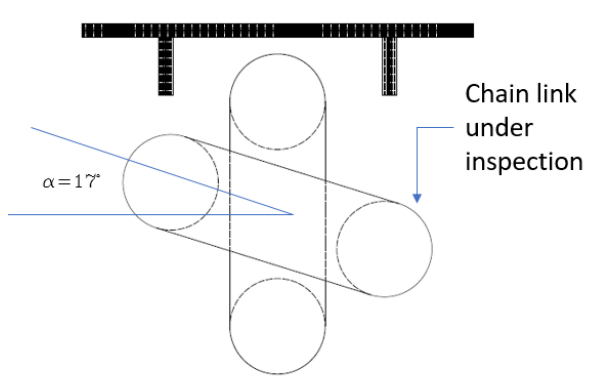

(c)

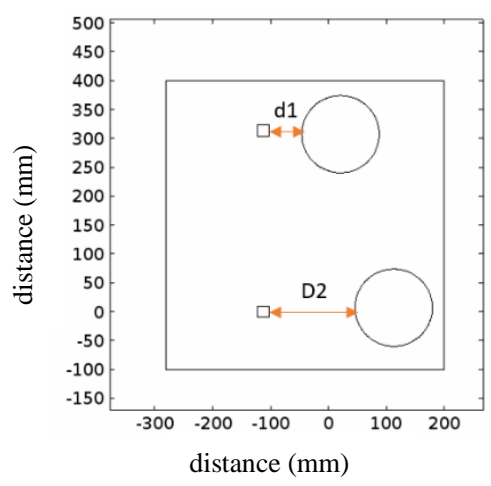

Figure 20: FEA Case 1-(a) 3D CAD design. (b)misaligned angle cross section. (c)simplified CAD

(a)

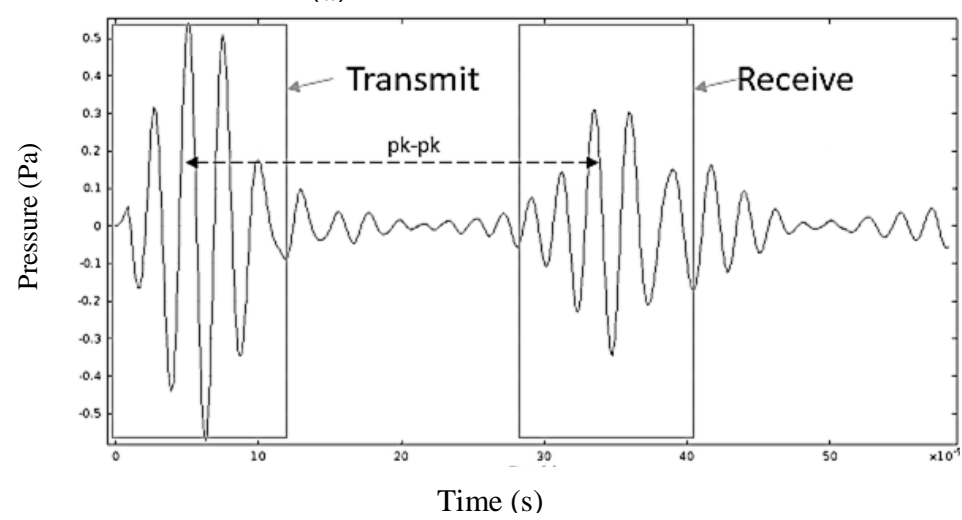

(b)

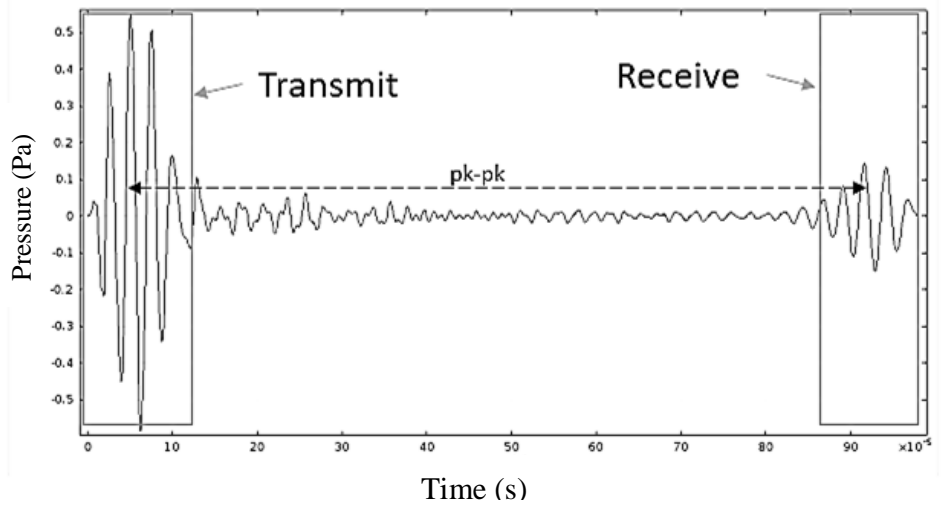

Figure 21 - Time-of-Flight signals. (a) from d1, (b) from d2

Output signals monitored in FEA case 1 are illustrated in figure 21 (d1- figure 21-a , d2- figure 21-b). Distance $\mathrm{d} 1$ and $\mathrm{d} 2$ were calculated by considering the peak to peak time of arrival (refer figure 21). d1 was calculated as $48.96 \mathrm{~mm}$. d2 was calculated as $148.09 \mathrm{~mm}$. Therefore, the 
misaligned angle was calculated by considering trigonometry between transducer positions and $\mathrm{d} 1, \mathrm{~d} 2$ distances. The calculated misaligned angle is as follows,

$\alpha=\tan ^{-1}\left\{\frac{d 2-d 1}{L a}\right]=\left\{\frac{148.09-48.96}{314.2}\right]=17.51^{\circ}$

Where, La is known (fixed) distance between transducers. Sign of the angle can be used to determine the clockwise and counter clockwise directions. In order to understand the tilt type misalignment, the flowing setup was proposed (refer figure 22).

(a)

Transducer

attachment frame


(c)

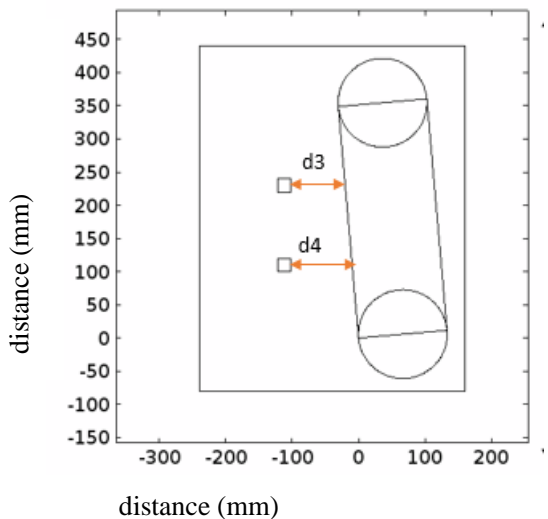

Figure 22: FEA Case 2-(a) 3D CAD design. (b)misaligned angle cross section. (C)simplified CAD

(a)

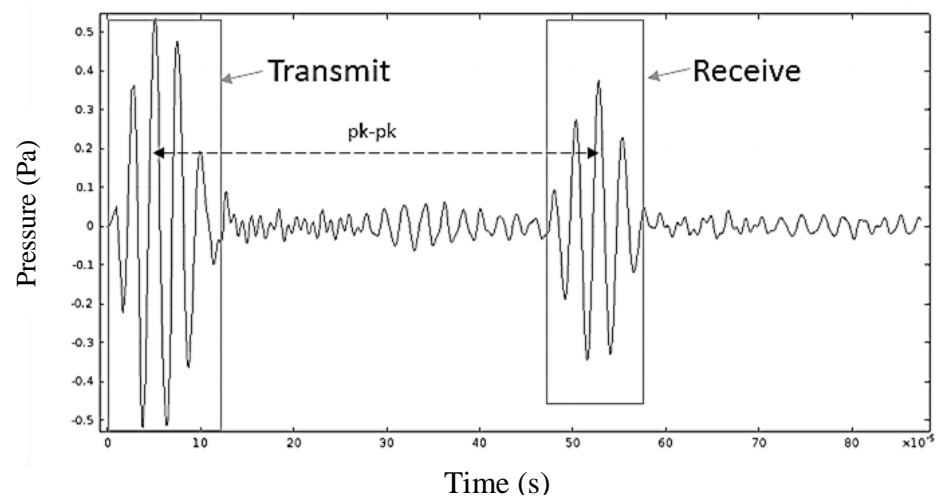

(b)

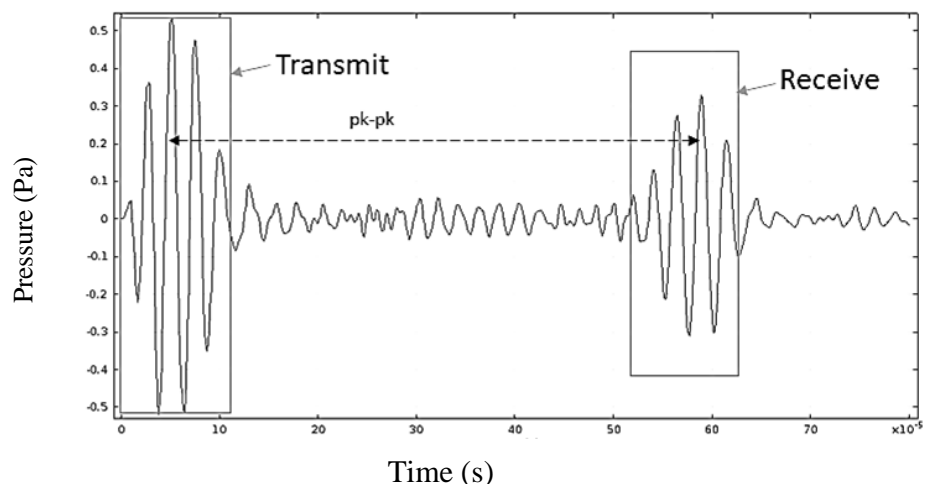

Figure 23 - Time-of-Flight signals. (a) from d3, (b) from d4

Output signals monitored in FEA case 2 are illustrated in figure 23 (d3- figure 23-a, d4- figure 23-b). Distances d3 and d4 were calculated by considering the peak to peak time (refer to figure 23). $\mathrm{d} 3$ was calculated as $47.55 \mathrm{~mm}$. $\mathrm{d} 4$ was calculated as $92.18 \mathrm{~mm}$. Therefore, the misaligned angle was calculated by considering trigonometry between transducer positions and $\mathrm{d} 3, \mathrm{~d} 4$ distances. The misaligned angle is as follows, 
$\varnothing=\tan ^{-1}\left\{\frac{d 4-d 3}{L b}\right]=\left\{\frac{92.18-47.55}{120}\right]=5.06^{\circ}$

Where, Lb is the known (fixed) distance between transducers. The sign of the angle can be used to determine the clockwise and counter clockwise directions.

According to the above examples, it is possible to use ultrasound distance measurement to estimate the misalignment angle of the chain links.

Table 1- parametric data used in the numerical modelling

\begin{tabular}{|r|l|}
\hline Parameter / expression & Value \\
Frequency $(f)$ & $40 \mathrm{kHz}$ \\
Number of cycles $(n)$ & 5 \\
Angular frequency $(w)$ & $2.5133 \mathrm{E} 5 \mathrm{~Hz}$ \\
Speed of sound in air $(c)$ & $343 \mathrm{~m} / \mathrm{s}$ \\
Density of air (app at sea level) & $1.225 \mathrm{kgm} \mathrm{m}^{-3}$ \\
Density of iron & $7700 \mathrm{kgm}{ }^{-3}$ \\
Speed of sound in iron & $5130 \mathrm{~m} / \mathrm{s}$ \\
Maximum element size & $8.5750 \mathrm{E}-4 \mathrm{~m}$ \\
Sampling frequency & $2.5 \mathrm{E}-5 \mathrm{~s}$ \\
Maximum element size & $0.8 \mathrm{~mm}$ \\
Minimum element size & $0.015 \mathrm{~mm}$ \\
Mesh type & Free triangular mesh \\
\hline
\end{tabular}

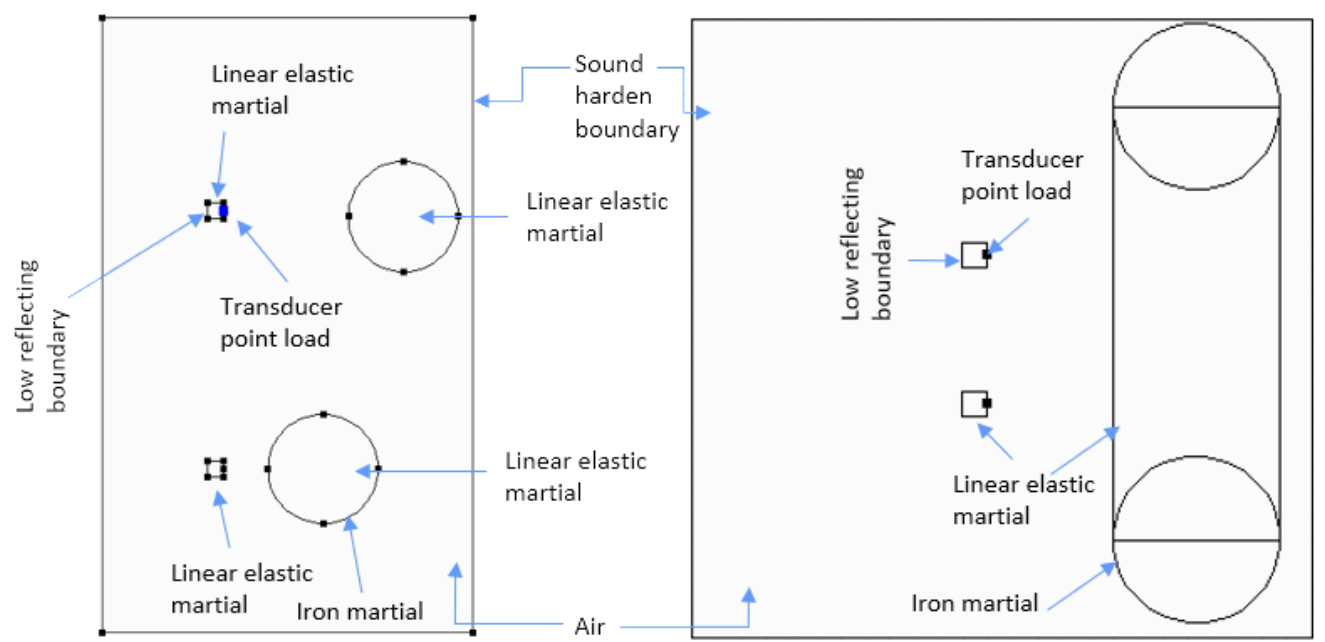

Figure 24- COMSOL FEA model lay out and boundaries 


\section{Conclusions}

This study was able to upgrade a previously designed magnetic adhesion tracked-wheel mooring chain climbing robot to address the misalignment issues of operational mooring chains. The previous version of the climbing robot was unable to demonstrate climbing when there is a misalignment presented in the chain. Two types of misalignments (chain twist and chain tilt) were studied and a mathematical model of a robot leg was proposed. Then the proposed model was modified according to the mechanical needs of the climbing robot. The prototyped robot leg was introduced to a single tracked-wheel module of the previous robot and experimental studies were carried out. The complete robot system was tested on a threelink mooring chain segment to study the climbing capability. For this study, 5 to $17^{\circ}$ of link twist and 1 to $5^{\circ}$ link tilt misalignments were introduced to the chain link, and the robot was able to adapt the tracked-wheel unit by using the newly added Cartesian robot leg. As a result of this study, the idea of an orthogonally placed Cartesian legged-magnetic adhesion tracked wheel robotic platform which can eliminate concerns related to the misaligned mooring chain climbing has been established.

\section{Acknowledgement}

This research work has been funded by the London South Bank Innovation Centre (LSBIC), the National Structural Integrity Research Centre (NSIRC) and TWI (NDT department), Cambridge. The authors express their sincere gratitude to the staff of LSBIC, TWI (NDT section) and Dr Jonathan Mark Selig for their support. The work is supported by InnovateUK with grant 102905 RIMCAW.

\section{References}

[1]. Angulo, Á., Edwards, G., Soua, S. \& Gan, T.-H., 2017. Mooring Integrity Management: Novel Approaches Towards In Situ Monitoring. In: Structural Health Monitoring - Measurement Methods and Practical Applications. s.1.:Intechopen, pp. 87-108.

[2]. Edwards, G. R. et al., 2013. www.moorinspect.eu. [Online] Available at: 
http://www.moorinspect.eu/publications/NDT2012MoorInspect_latest.pdf [Accessed 21 December 2015].

[3]. Elman, P., Bramande, . J., Elletson, . E. \& Pinheiro, K., 2013. Reducing Uncertainty Through the Use of Mooring Line Monitoring. Rio de Janeiro, Brazil, s.n.

[4]. García, J. L. et al., 2004. Automated Off-shore studless chain inspection system. Montreal, Canada, s.n.

[5]. Gordon, R. B., Brown, M. G., Allen, E. M. \& DNV GL, 2014. Mooring Integrity Management: A State-of-the-Art Review. Houston, Texas, Offshore Technology Conference.

[6]. Hall, A. D., Welaptega Marine Limited \& Lethbridge, . G. M., 1999. Digital Imaging for Subsea Operations: Innovative ROV Tooling and Image Analysis Combinations for Mooring Chain Assessments Yield Cost Savings and Operational Confidence for Offshore Operators. Aberdeen, Scotland, Offshore Europe Oil and Gas Exhibition and Conference.

[7]. Noble Denton Europe Limited, 2016. Floating production system -JIP FPS mooring integrity, Aberdeen: Health And Safety Executive.

[8]. Rudlin, J., 2014. Multi-channel ultrasonic inspection of a mooring chain for fatigue cracks. Prague, Czech Republic, s.n.

[9]. Ruiz, A. G., Sattar, T. P., Sanz, . C. M. \& Rodriguez-Filloy, B. S., 2014. Inspection of floating platform mooring chain with a climbing robot. Poznań, Poland, s.n.

[10]. Weiss, P., Andritsos, F., Schom, F. \& Fidani, A., 2004. Innovative Robotic Solutions for the Survey and Certification of Ships and Mobile Offshore Units. Siguenza, Spain, s.n.

[11]. Dissanayake, M., Sattar, T., Pinson, I. \& Gan, T.-H., 2017. Tracked-wheel crawler robot for vertically aligned mooring chain climbing design. Kandy, Sri Lanka, s.n.

[12]. M. Dissanayake, T. Sattar, G. Tat-Hean, I. Pinson and S. Lowe, 2018. Design and prototype of a magnetic adhesion tracked-wheel robotic platform for mooring chain inspection. Journal of Systems and Control Engineering:Proceedings of the Institution of Mechanical Engineers, Part I, Issue doi:10.1177/0959651818774479. 
[13]. Kai-tung Ma, Shu, H., Philip , S. \& Arun , D., 2013. A Historical Review on Integrity Issues of Permanent Mooring Systems. Houston, Texas, s.n.

[14]. Welapetage, 2017. http://www.welaptega.com/. [Online]

Available at: http://www.welaptega.com/services/subseameasurement/

[Accessed 2506 2018].

[15]. Williams , S., 2008. cordis.europa.eu. [Online]

Available at: http://cordis.europa.eu/docs/publications/1216/121625181-6_en.pdf

[Accessed 10 December 2015].

[16]. Yoshie, M. \& Toshinari , T., 2013. Field test of a practical test model of maintenance examination system for mooring facilities and additional installation of chain grasping frame in the vehicle. Tokyo, Japan, s.n. 